unpolarised radiation passing parallel and at right angles to the axis and have been fortunate in having available, by the courtesy of Messrs. A. Hilger Ltd., unusually large thicknesses of the material. The differences in both intensity and structure of the bands for the two directions are striking as may be seen from Fig. 1. The figure also shows the curve of galvanometer deflections (III) using a small 'hohlraum' as source. The bands recorded by Dreisch for crystalline quartz parallel to the axis appear in this curve and are due to the prism, which was of quartz. There is an apparent wave-length discrepancy between curves I and III, because I is referred to a horizontal datum and III to an energy curve falling fairly steeply to the right. This causes an apparent displacement of the bands in III to the right.

It appears, from curve $\mathrm{I}$, that the ordinary ray is completely absorbed between $2 \cdot 83 \mu$ and $3.02 \mu$ in $63 \mathrm{~mm}$. of quartz. In the $83 \mathrm{~mm}$. used for curve II there is nevertheless a measurable fraction of radia-

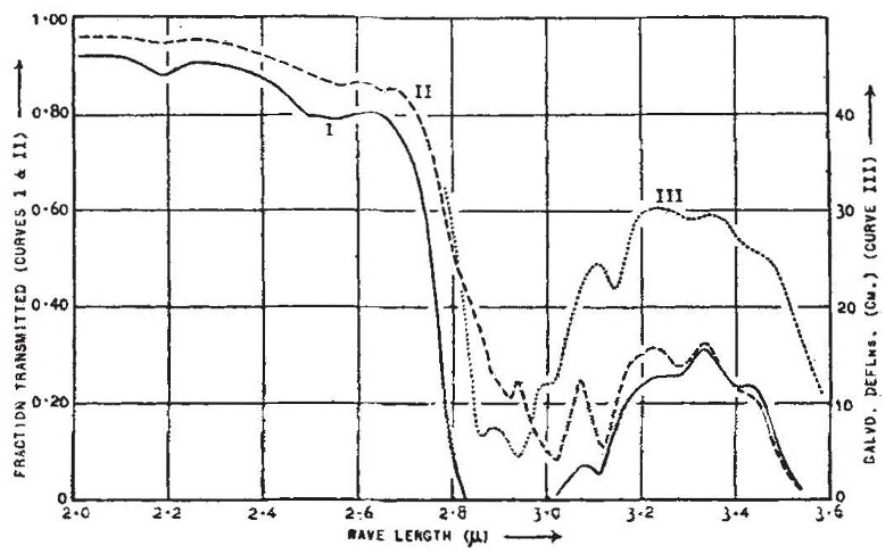

Fia. 1.-Infra-red absorption of quartz. I. Path $63 \mathrm{~mm}$. parallel to axis. II. Path $83 \mathrm{~mm}$. perpendicular to plane of optic and electric axes. III Galvanometer deflections without specimen.

tion transmitted, which must be ascribed to the extraordinary ray. Allowing for a 50 per cent initial loss from the beam by absorption of the ordinary ray, it follows that the general intensity of absorption of the extraordinary ray must be considerably less than that of the ordinary ray in this region. Further, a comparison of curves II and III suggests that the band structures will prove different. The wave. length of maximum absorption of a band will therefore vary with the direction and state of polarisation of the beam in the quartz. The differences extend some distance on each side of the region mentioned, although here they are not so directly interpreted, and it is interesting to note that Barnes ${ }^{4}$ has recently obtained indications of similar dichroism in the band at $38 \mu$ in the far infra-red.

In curve I the band at $3 \cdot 11 \mu$ is, I think, to be identified with Dreisch's fourth band, the wavelength of which he does not state. The bands at $2 \cdot 19$, $3 \cdot 27$, and $3 \cdot 41 \mu$ as well as some structure between 2.5 and $2.7 \mu$ have not, so far as I know, been recorded before. I have also found a band in fused silica at $2 \cdot 23 \mu$ which is of a similar order of intensity to that at $2 \cdot 19 \mu$ above. This is interesting in view of Barnes's results (loc. cit.) indicating bands in fused silica in the far infra-red which, it is suggested, are in some way related to bands occurring in crystalline quartz at rather shorter wave-lengths.
Further work on this subject has been unavoidably held up for some months but I hope shortly to carry out a more extensive investigation of the effects using polarised radiation.

Physics Department, Armstrong College.

(Newcastle-on-Tyne),

Durham University. Oet. 17

Ann. Phys., 55, 49; 1895.

Ann. Phys. 61,$687 ; 1897$

“ Phys, Rev, 39, $562 ; 1932$

\section{Electrical Ignition of Explosive Gaseous Mixtures}

Prof. TAYLOR-Jones, in discussing the thermal theory of the electrical ignition of explosive gaseous mixtures, states that "There is nothing in the thermal theory . . . to suggest that the energy of the translational motion of the molecules, or that of their rotational or vibratory motion plays a pre. ponderating part in the process of ignition" ("Induction Coil Theory and Applications", Pitman, London, 1932). Whilst in complete accord with the first part (up to and including "theory") I cannot agree with this statement as a whole, for the following reasons :

The thermal theory of electrical ignition has been put forward by Taylor-Jones, Morgan and Wheeler ${ }^{1}$ in terms stating that "the ignition of a gaseous mixture depends primarily ... on the heating of a sufficient volume to a sufficient temperature". But this view must involve the supposition that internal molecular energy plays no rôle in ignition, because it is well known that molecules can be excited without at the same time necessarily increasing their translational energy. Thus Taylor-Jones's statement as quoted above should be amended to read "The thermal theory asserts that only the translational energy of the molecules plays a part in ignition", because it cannot be supposed that the ratio between excited and normal molecules in a gas is determined solely and in all circumstances by the temperature.

There are, however, many well-established facts, such as, for example, the photo-ignition of hydrogenchlorine mixtures, which suggest that internal molecular energy does in fact play a preponderating rôle in ignition and thus appear to contradict the view of the importance assigned by the thermal theory to translational energy. Further, in conjunction with H. H. Thompson" I have shown by a crucial experiment that fact and the thermal theory are in direct conflict.

\section{G. Ingle Finch.}

Imperial College of Science and Technology, South Kensington, London, S.W.7. Nov. 22.

1 Phil. Mag., 43, 359.

2 Proc, Roy, Soc., A, 134, 343

\section{Surface Tension near the Gritical Point}

IT is well known that when measured values of surface tension are plotted against the temperature a nearly linear law is obtained, but that as a rule the curve is concave upwards and approaches the critical point almost tangentially (even if not quite so) the 